\title{
A big thank you to our reviewers in the past year!
}

Good reviewers are essential to the success of any journal and peer review is a major pillar of science. We are grateful to those mentioned below to have dedicated their time and expertise to help our authors improve and refine their manuscripts and support the Editor(s) in the decision making process in the past year. The following received our Top Reviewer of the Year award: Yousuke Nakai, Amol Bapaye, Rapat Pittayanon, Andrea Tringali. We wish all our readers, reviewers, authors and friends a happy and prosperous 2021 !

\begin{tabular}{|c|c|c|c|}
\hline Aabakken, Lars & Bonavina, Luigi & Dougherty, Michael & Hamada, Kenta \\
\hline Aadam, A. Aziz & Bonnichsen, Mark & Draganov, Peter & Han, Joung-Ho \\
\hline Abe, Seiichiro & Bonura, Giuliano & Dumitru, Eugen & Hanaoka, Noboru \\
\hline Abe, Yasuhiko & Boskoski, Ivo & Dumonceau, Jean-Marc & Hara, Kazuo \\
\hline Adler, Andreas & Brathwaite, Collin & Dumoulin, Franz Ludwig & Hashimoto, Satoru \\
\hline Agapov, Mikhail & Brewer Gutierrez, Olaya I. & & Hashimoto, Taiki \\
\hline Ageykina, Natalia & Buda, Andrea & Ebigbo, Alanna & Hassan, Cesare \\
\hline Aghaie Meybodi, Mohamad & Bugajski, Marek & Eckardt, Alexander & Hayashi, Yoshikazu \\
\hline Aihara, Hiroyuki & Bustamante, Marco & El Hajjar, Ahmad & Haycock, Adam \\
\hline Akahoshi, Kazuya & & Elahmedi, Mohamed O & Hedenstrom, Per \\
\hline Akasaka, Tomofumi & Cadoni, Sergio & Elhendawy, Mohammed & Hikichi, Takuto \\
\hline Akimoto, Teppei & Cai, Qiang & Eliakim Rami & Hirasawa, Kingo \\
\hline Albéniz, Eduardo & Caire, M. & Elli, Luca & Hirasawa, Toshiaki \\
\hline Amano, Yuji & Camacho, Santiago & Esaki, Mitsuru & Histace, Aymeric \\
\hline Amit, Bhatt & Candemir, Sema & Esposito, Gianluca & Ho, Shiaw-Hooi \\
\hline Anderloni, Andrea & Carrara, Silvia & Esteban, Jose-Miguel & Hoare, Jonathan M. \\
\hline Ang, Tiing Leong & Castro, Fernando & Etoh, Tsuyoshi & Hochberger, Juergen \\
\hline $\begin{array}{l}\text { Angsuwatcharakon, } \\
\text { Phonthep }\end{array}$ & $\begin{array}{l}\text { Cave, David } \\
\text { Chaussade, Stanislas }\end{array}$ & Eusebi, Leonardo & $\begin{array}{l}\text { Holme, Øyvind } \\
\text { Hookey, Lawrence C }\end{array}$ \\
\hline Antonelli, Giulio & Chavan, Radhika & Facciorusso, Antonio & Hori, Keisuke \\
\hline Antonini, Filippo & Chen, Shiyao & Familiari, Pietro & Hoteya, Shu \\
\hline Arai, Makoto & Cheng, Spencer & Fayad, Lea & Hotta, Kinichi \\
\hline Arcidiacono, Paolo Giorgio & Cheon, Young Koog & Filmann, Natalie & Hou, Ming-Chih \\
\hline Arvanitakis, Marianna & Chiu, Han-Mo & Freeman, Martin & Hsieh, Yu-Hsi \\
\hline Asokkumar, Ravishankar & Chiu, Philip & Fry, Lucia & Hu, Bing \\
\hline Averbach, Marcelo & Cho, Chang Min & Fujii-Lau, Larissa & Hu, Jianwei \\
\hline \multirow[t]{2}{*}{ Aziz, Muhammad } & Cho, Yu Kyung & Fujimoto, Ai & Hüneburg, Robert \\
\hline & Chukmaitov, Askar & Fujishiro, Mitsuhiro & Hyun, Jong Jin \\
\hline Bai, Yu & Coron, Emmanuel & Fukuda, Hiromu & \\
\hline Baldaque-Silva, Francisco & Cotter, José & Fukuzawa, Masakatsu & leiri, Satoshi \\
\hline Bansal, Rinkesh & Crinó, Stefano Francesco & Fusaroli, Pietro & Ikura, Yoshihiro \\
\hline Bapaye, Amol & & & Imaeda, Hiroyuki \\
\hline Baron, Todd & Daizen, Hirata & Galvão, Manoel & Imagawa, Atsushi \\
\hline Barret, Maximilien & Dawod, Enad & Garborg, Kjetil & Imai, Kenichiro \\
\hline Bartel, Michael & De La Mora-Levy, Jose G. & Gervasoni, Mauricio & Inoue, Tadahisa \\
\hline Barthet, Marc & de Lange, Thomas & Glover, Ben & Inoue, Takahiro \\
\hline Barua, Ishita & de Moura, Eduardo & Goda, Kenichi & Irani, Shayan \\
\hline Basford, Peter John & de Nucci, Germana & Gonda, Tamas & Isayama, Hiroyuki \\
\hline Bauerfeind, Peter & Deprez, Pierre & González Suárez, Begoña & Ishida, Tsukasa \\
\hline Bautista, Inmaculada & Desai, Pankaj & Gordon, Stuart & Ishii, Naoki \\
\hline Bazerbachi, Fateh & Dhir, Vinay & Goto, Osamu & Ito, Sayo \\
\hline Belle, Sebastian & Diaconescu, Smaranda & Greenwald, Bruce & Iwagami, Hiroyoshi \\
\hline Belletrutti, Paul & Diehl, David & Grimes, Kevin & Iwashita, Takuji \\
\hline Berger, Thomas & Dijmarescu, Irina & Grover, Samir & \\
\hline Bertani, Helga & Dinis-Ribeiro, Mário & Guaraldi, Simone & Jackson, Christian \\
\hline Blancas Valencia, Juan Manuel & Dobashi, Akira & Guda, Nalini & Jacques, Jérémie \\
\hline Boal Carvalho, Pedro & Dolwani, Sunil & Guedes, Hugo & Jaime, Francisca \\
\hline
\end{tabular}


Jeong, Seok

Jimenez-Garcia, Victoria

Jin, Zhezhen

Kadota, Tomohiro

Kaffes, Arthur

Kaltenbach, Tonya

Kanemitsu, Takao

Kanesaka, Takashi

Kanno, Yoshihide

Kantsevoy, Sergey

Kanzaki, Hiromitsu

Karstensen, John

Kashima, Shin

Kato, Motohiko

Kawachi, Hiroshi

Kawai, Manabu

Kawakubo, Kazumichi

Kawamura, Masashi

Kawamura, Takuji

Kazuo, Ohtsuka

Kikuchi, Daisuke

Kim, Hyun Gun

Kim, Tae Hyeon

Kishino, Takaaki

Kitamura, Katsuya

Kitano, Masayuki

Klapman, Jason

Kobara, Hideki

Kobayashi, Nozomu

Koch, Arjun

Kogure, Hirofumi

Koike, Tomoyuki

Komeda, Yoriaki

Kongkam, Pradermchai

Kono, Yoshiyasu

Kothari, Shivangi

Kotwal, Vikram S.

Kozarek, Richard

Krishna, Somashekar

Kröner, Paul

Kudo, Toyoki

Kumbhari, Vivek

Kuraoka, Naosuke

Kusano, Chika

Kushima, Ryoji

Kuwai, Toshio

Kuwatani, Masaki

Kylanpaa, Leena

Laiyemo, Adeyinka O. Lakhtakia, Sundeep

Laquiere, Arthur

Lee, Ching-Tai

Lee, Sang Soo

Lee, Yun Nah

Leighton, Jonathan

\begin{tabular}{|c|c|}
\hline Lemmers, Arnaud & Nonaka, Kouichi \\
\hline Libânio, Diogo & Nonaka, Satoru \\
\hline \multicolumn{2}{|l|}{ Lipham, John C. } \\
\hline Lisotti, Andrea & Oakland, Kathryn \\
\hline Liu, Deliang & O’Connor, Sam \\
\hline Lo, Simon & Ofstead, Cori \\
\hline Lopez-Nava, Gontrand & Ogura, Takeshi \\
\hline Lorenzo, Michele & Ohata, Ken \\
\hline \multirow[t]{2}{*}{ Louis, Hubert } & Ohmori, Masayasu \\
\hline & Ono, Satoshi \\
\hline Macedo, Guilherme & Ono, Yoichiro \\
\hline Maehata, Tadateru & Oppong, Kofi \\
\hline Maekawa, Akira & Osawa, Satoshi \\
\hline Maekita, Takao & Othman, Mohamed O. \\
\hline Mahadev, Srihari & Oung, Borathchakra \\
\hline Manes, Gianpiero & Ozuner, Gokhan \\
\hline \multicolumn{2}{|l|}{ Man-i, Mariko } \\
\hline Mariani, Alberto & Paik, Woo Hyun \\
\hline Martinek, Jan & Palazzo, Laurent \\
\hline Matharoo, Manmeet & Panzuto, Francesco \\
\hline Mathew, Philip & Papalois, Apostolos \\
\hline McGrath, Kevin & Parra-Blanco, Adolfo \\
\hline Mehta, Nilay & Pasha, Shabana \\
\hline Meining, Alexander & Paspatis, Gregorios \\
\hline Mekaroonkamol, Parit & Pech, Oliver \\
\hline Michida, Tomoki & Pennazio, Marco \\
\hline Minami, Hitomi & Pereira, Stephen \\
\hline Mion, Francois & Perez-Miranda, Manuel \\
\hline Misra, Sri & Perumpail, Ryan \\
\hline Miyahara, Ryoji & Pham, Khanh Do-Cong \\
\hline Moenkemueller, Klaus & Piessevaux, Hubert \\
\hline Moise, Alida & Pineau, Lionel \\
\hline Morais, Rui & Pinho, Rolando \\
\hline Moreels, Tom & Pittayanon, Rapat \\
\hline Mori, Yuichi & Pizcoya, Alejandro \\
\hline Moriyama, Tomohiko & Poincloux, Laurent \\
\hline Moura, Diogo & Poley, Jan-Werner \\
\hline Moyer, Matthew & Pontone, Stefano \\
\hline Mukai, Shuntaro & Portillo, Isabel \\
\hline Mukkada, Roy & Pouw, Roos \\
\hline \multirow[t]{2}{*}{ Murakami, Takashi } & Pouw, Roos E. \\
\hline & Probst, Andreas \\
\hline Nabi, Zaheer & Puri, Amarender \\
\hline Naganuma, Makoto & Puri, Rajesh \\
\hline \multicolumn{2}{|l|}{ Naito, Yuji } \\
\hline Naitoh, Itaru & Ragunath, Krish \\
\hline Nakahara, Kazunari & Rai, Praveer \\
\hline Nakai, Yousuke & Raijman, I. \\
\hline Nakanishi, Hiroyoshi & Ramchandani, Mohan \\
\hline Napoleon, Bertrand & Rana, Surinder \\
\hline Naqvi, Syed & Rath, Timo \\
\hline Navaneethan, Udayakumar & Ravindran, Srivathsan \\
\hline Ngamruengphong, Saowanee & Reddy, Nageshwar \\
\hline Ngu, Wee Sing & Regula, Jaroslaw \\
\hline Niikura, Ryota & Rerknimitr, Rungsun \\
\hline Nishida, Tsutomu & Ribeiro, Igor \\
\hline Nishizawa, Toshihiro & Ridtitid, Wiriyaporn \\
\hline
\end{tabular}

Rimmele, Thomas

Rondonotti, Emanuele

Rönnow, Carl-Fredrik

Rosa, Bruno

Rustagi, Tarun

Ryou, Marvin

Ryu, Ji Kon

Safatle-Ribeiro, Adriana Vaz

Saito, Hirokazu

Saito, Yutaka

Saltzman, Joel

Sanaka, Madhusudhan R.

Sandru, Vasile

Sarkar, Sanchoy

Sasaki, Takashi

Saurin, Jean-Christophe

Sawatzki, Mikael

Saxena, Payal

Senore, Carlo

Sethi, Amrita

Sey, Michael

Shami, Vanessa

Shibagaki, Koutarou

Shichijo, Satoki

Shimamura, Yuto

Shinmura, Kensuke

Shinozaki, Satoshi

Shitani, Masahiro

Shiwaku, Hironari

Siau, keith

Siddiqui, Uzma D.

Singh, Shailendra

Singla, Vikas

Song, Tae Jun

Sonnenberg, A

Spada, Cristiano

Spadaccini, Marco

Sperti, Cosimo

Strand, Daniel

Sugimoto, Mitsushige

Sumiyama, Kazuki

Suzuki, Haruhisa

Suzuki, Noriko

Suzuki, Sho

Takabayashi, Kaoru

Takagaki, Nobumasa

Takamaru, Hiroyuki

Takenaka, Mamoru

Takeuchi, Yoji

Takizawa, Kohei

Tamai, Naoto

Tanaka, Kyosuke

Tanaka, Shinji

Tanaka, Shinwa

Tashima, Tomoaki 
Teoh, Anthony

Teshima, Christopher

Testoni, Pier Alberto

Thomas-Gibson, Siwan

Thosani, Nirav

Thoufeeq, Mo

Tiyarattanachai, Thodsawit

Toh Yoon, Ezekiel Wong

Toyonaga , Takashi

Toyoshima, Osamu

Triantafyllou, Konstantinos

Tribonias, Georgios

Trindade, Arvind

Tringali, Andrea
Tsuji, Yosuke

Tyberg, Amy

Tziatzios, Georgios

Uedo, Noriya

Ujiki, Michael

Urabe, Yuji

Uraoka, Toshio

Vanderlaan, Paul

Vergis, Nikhil

Viale, Edi

Villa, Edward

Voiosu, Theodor
Wagh, Mihir

Wang, Xiao

Weigt, Jochen

Weston, Brian

Wilcox, C

Willingham, Field

Yabuuchi, Yohei

Yamada, Masayoshi

Yamaguchi, Daisuke

Yamamoto, Yorimasa

Yamao, Kentaro

Yamasaki, Yasushi

Yamashina, Takeshi
Yano, Tomonori

Yip, Hon Chi

Yoshida, Masao

Yoshida, Naohiro

Yoshida, Naohisa

Yoshio, Toshiyuki

Zhu, Bo-Qun

Zorrón Cheng Tao Pu,

Leonardo 